\title{
IMPLANTAÇÃO DO CCL - CENTRO DE CONTROLE LOGÍSTICO COMO FERRAMENTA DE APOIO À GESTÃO DO TRANSPORTE RODOVIÁRIO DE MINÉRIO DE FERRO NA MINERAÇÃO DA GERDAU*
}

Guilherme Anselmo Guimarães dos Santos ${ }^{1}$ Ulisses Van Zanten Mendes ${ }^{2}$ Gustavo Henrique Silva Cunha ${ }^{3}$ Guilherme Lage Becker ${ }^{4}$ José Carlos Guimarães ${ }^{5}$

\section{Resumo}

O presente trabalho buscou apresentar os resultados e benefícios oriundos da implantação de uma ferramenta de gestão logística denominada CCL - Centro de Controle Logístico na operação logística do minério de ferro na Gerdau, que por sua vez é caracterizada pela movimentação utilizando predominantemente o transporte rodoviário. Os resultados do trabalho apontam para obtenção da eficiência da gestão logística na operação de minério de ferro, mediante integração de sistemas de informação a uma central que consolida todos os dados gerando informações, ora em tempo real permitindo tomadas de decisão imediata, intervenção na operação e alteração de prioridades e, ora através da geração de $K P / s$ permitindo uma visão global do sistema logístico que engloba a operação em referência. Além disso, os painéis gerenciais oferecidos a partir do CCL são chaves no controle e gestão do fluxo logístico da operação. Por fim, um dos maiores ganhos obtidos é o controle total da operação de transportes rodoviário, que por sua vez é realizada através de transportadores contratados, onde a Gerdau passou a ter acesso à visão geral dos aspectos operacionais e frota disponibilizada.

Palavras-chave: Gestão logística; Ferramentas gerenciais; Transporte rodoviário; Avaliação de desempenho.

\section{IMPLEMENTATION OF THE LCC - LOGISTICS CONTROL CENTER AS A TOOL TO SUPPORT MANAGEMENT OF ROAD TRANSPORTATION OF IRON ORE IN GMA - GERDAU AMERICAS MINING}

\section{Abstract}

This paper presented the results and benefits obtained with the implementation of a management tool called LCC - Logistics Control Center in the iron ore logistics operation at Gerdau, which in turn is characterized by the drive using predominantly road transportation. The results point to obtain the efficiency of logistics management in iron ore operation, through integration of information systems to a central consolidating all data generating information, sometimes in real time allowing taken an immediate decision, intervention in the operation and change priorities and, sometimes by generating KPIs allowing an overview of the logistics system which includes the operation in question. Furthermore, the management panels offered from the CCL are key in controlling and managing the logistics of the operation flow. Finally, one of the biggest gains is obtained full control of the road transport operation, which in turn is accomplished through contracted carriers, in which the Company has gained access to the overview of the operational aspects and available fleet.

Keywords: Logistics management; Management tools; Road transportation; Performance evaluation.

Mestre em Engenharia de Transportes pela COPPE/UFRJ, Graduação em Administração em ênfase em Comércio Exterior pela PUC-MG, Assessor Técnico de Logística, Gerência de Logística Operacional Mfe, Gerdau S/A, Ouro Preto, MG, Brasil.

2 MBA em Gestão de Negócios pelo IBMEC, Graduação em Administração com ênfase em Marketing pela Newton Paiva, Chefe de Logística Externa, Gerência de Logística Operacional Mfe, Gerdau S/A, Ouro Preto, MG, Brasil.

3 Graduação em Engenharia de Produção Civil pela FUMEC, Chefe de Gestão e Controle Logístico, Gerência de Logística Operacional Mfe, Gerdau S/A, Ouro Preto, MG, Brasil.

4 MBA Gestão Empresarial pela FDC, Graduação em Engenharia de Controle e Automação pela UFMG, Chefe de Logística de Entregas, Gerência de Logística Operacional Mfe, Gerdau S/A, Ouro Preto, MG, Brasil.

5 Graduação em Administração com ênfase em Comércio Exterior pela PUC-MG, Chefe de Logística de Entregas, Gerência de Logística Operacional Mfe, Gerdau S/A, Ouro Preto, MG, Brasil. 


\section{INTRODUÇÃO}

Um dos pilares na gestão da logística, está sem dúvidas, na qualidade das informações geradas concomitante às suas atividades operacionais. Neste contexto, a utilização de ferramentas e sistemas que oferecem precisão nas informações, bem como disponibilidade contínua e on-line, tem possibilitado um aumento na velocidade de tomada de decisões nas empresas [1].

O transporte rodoviário, por sua vez, é um dos pilares da logística no Brasil do ponto de vista de distribuição modal das cargas, principalmente em cargas de alto volume de movimentação como minério de ferro e produtos siderúrgicos. Seja no abastecimento direto dos pontos de consumo ou abastecimento de pontos intermediários como terminais de integração intermodal, sempre com o objetivo de otimizar sua eficiência operacional bem como cumprir os prazos e volumes.

Esse direcionamento, vem de encontro com as mudanças constantes no sistema de gestão de players do setor siderúrgico, através principalmente de sistemas de avaliação de desempenho, seja em operações próprias ou terceirizadas, onde dentre os quais encontram-se os transportadores rodoviários [2].

Dentre os projetos de gestão e tecnologia da informação de maior impacto e relevância deste setor, pode-se destacar o gerenciamento de frotas por meio de rastreamento por satélite, que se integrados aos conceitos de gestão logística e segurança se torna uma ótima ferramenta para gerenciamento da rotina do dia-a-dia nas operações de abastecimento e distribuição dos produtos movimentados no transporte rodoviário [3].

No presente trabalho, é apresentado o caso da operação de movimentação de minério de ferro próprio da GMA - Gerdau Mining Americas, negócio mineração da Gerdau, nos processos de abastecimento à Usina Ouro Branco de propriedade da própria Gerdau S/A bem como na distribuição do minério para vendas, tanto no mercado interno quanto exportação. O caso retrata a implantação de uma ferramenta de gestão de frota por rastreamento de satélite, nas empresas prestadoras do serviço de transporte rodoviário de minério de ferro, que por sua vez é integrada a outros fluxos de informação e consolidado na forma de um Centro de Controle Logístico.

No Brasil, segundo Monico [4] e Prado et al [5] este tipo de ferramenta é usualmente utilizado para gerenciamento de riscos, motivados principalmente pelo aumento constante dos eventos de roubos e furtos de carga, onde são monitorados posicionamentos dos veículos e suas rotas. A Gerdau por sua vez, direcionou a ferramenta para os moldes utilizados nos EUA e Europa, onde o objetivo principal da ferramenta passa a ser o gerenciamento com foco em produtividade através da redução do tempo de ciclo, monitoramento dos pontos de carga e descarga on-line, disponibilidade de frota e segurança integrados à uma central de controle $[6,7]$.

Com isso, os prestadores de serviço de transporte rodoviário podem também contar com informações relevantes para o gerenciamento de suas operações, como produtividade de sua frota, alocação de ativos, otimização, entre outros, garantindo robustez do sistema para múltiplas funções dentro da operação de logística do minério de ferro. 


\section{MATERIAIS E MÉTODOS}

Para o desenvolvimento deste trabalho, foram considerados três agentes fundamentais:

- O transportador rodoviário de carga, sendo usuário primário da tecnologia de rastreamento e ponto central do processo;

- A Gerdau, também definida como embarcador, sendo usuário secundário do sistema de rastreamento que por sua vez absorve os benefícios e imperfeições das informações geradas pelo sistema de rastreamento;

- Os fornecedores da tecnologia utilizada para geração das informações que compõe o CCL.

O método utilizado para desenvolvimento do trabalho foi o estudo de caso na operação de logística da GMA no processo de abastecimento e distribuição de minério bruto e acabado.

O fluxograma das atividades desenvolvidas para a implantação do sistema é apresentado na figura 1, abaixo:

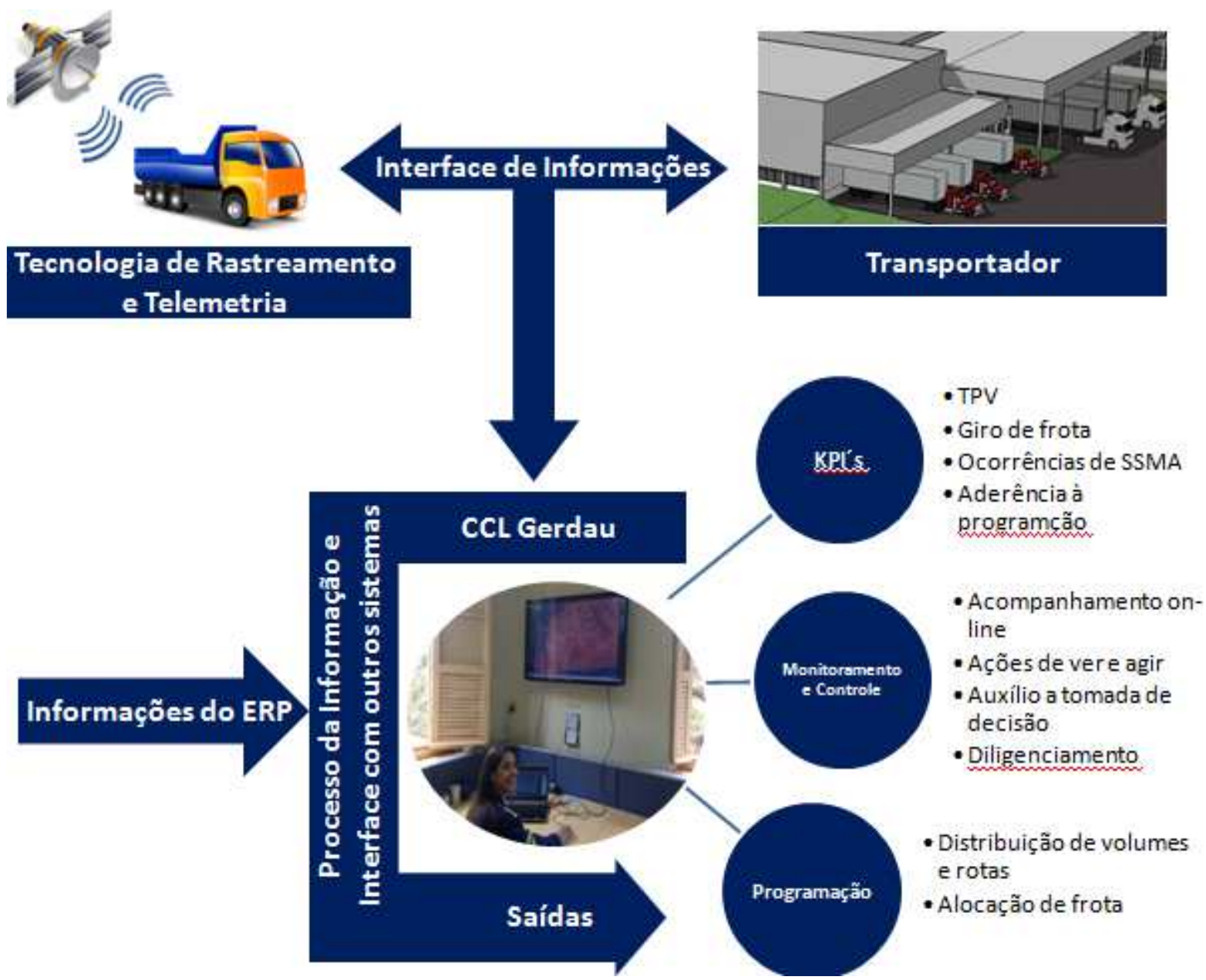

Figura 1 - Fluxograma das atividades na consolidação do CCL na GMA 


\section{RESULTADOS E DISCUSSÃO}

\subsection{Contextualização da Operação Logística de Minério de Ferro na Gerdau}

A operação de logística de minério de ferro na Gerdau tem papel crucial ao negócio uma vez que está sob sua responsabilidade toda a movimentação de matéria-prima entre minas, de produto para abastecimento à usina de Ouro Branco e entrega de produtos vendidos a terceiros; desde o processo de carregamento nas minas, pesagem, faturamento e operação de transporte (abastecimento e distribuição).

A operação de transporte, por sua vez, responsável pelas movimentações físicas entre os pontos de origem e consumo é realizada por transportadores contratados, que mantém uma frota regular operando nos fluxos e rotas do minério de ferro prédeterminados pela Gerdau.

Diante da complexidade do sistema de abastecimento e distribuição do minério de ferro nas operações da Gerdau, a logística tem sido constantemente desafiada a implantar ferramentas e ações que proporcionam melhoria contínua tanto no contexto operacional quanto no fluxo de informações, que por sua vez dão subsídio para a eficiência na gestão.

Neste sentido, foi realizado um projeto para implantação de um centro de controle, que consolida informações geradas em tempo real, durante as movimentações, e que por sua vez, se transformam em inputs para consolidação de outros indicadores tratados individualmente e coletivamente servindo para melhorar a eficiência na gestão de toda a operação logística.

\subsection{CCL no Contexto da Operação de Minério de Ferro na Gerdau}

$\mathrm{Na}$ implantação do CCL na operação de minério de ferro da Gerdau, foram realizadas as etapas apresentadas na figura 2, abaixo:

\begin{tabular}{|c|c|c|c|}
\hline Iniciação & Planejamento & Execução & Encerramento \\
\hline $\begin{array}{l}\checkmark \text { Levantamento das } \\
\text { demandas }\end{array}$ & $\begin{array}{l}\checkmark \text { Modelagem do } \\
\text { sistema }\end{array}$ & $\begin{array}{l}\checkmark \text { Inserção dos pontos } \\
\text { nodais no sistema }\end{array}$ & $\begin{array}{l}\checkmark \text { Avaliação dos } \\
\text { resultados }\end{array}$ \\
\hline \multirow{9}{*}{$\begin{array}{l}\checkmark \text { Conceituação do } \\
\text { sistema } \\
\checkmark \text { Premissas } \\
\checkmark \text { Restrições }\end{array}$} & $\begin{array}{l}\checkmark \text { Escolha da } \\
\text { tecnologia }\end{array}$ & \multirow{2}{*}{$\begin{array}{l}\checkmark \text { Construção das } \\
\text { macrocercas e } \\
\text { microcercas }\end{array}$} & \multirow[t]{9}{*}{$\begin{array}{l}\checkmark \text { Implementação / } \\
\text { rotina }\end{array}$} \\
\hline & $\checkmark$ Desenho da & & \\
\hline & operação & \multirow{2}{*}{$\begin{array}{l}\checkmark \text { Customização do } \\
\text { sistema para } \\
\text { múltiplas funções }\end{array}$} & \\
\hline & $\checkmark$ Definir os $\mathrm{KPI}$ 's & & \\
\hline & $\begin{array}{l}\checkmark \text { Estruturação dos } \\
\text { relatórios }\end{array}$ & \multirow{2}{*}{$\begin{array}{l}\checkmark \text { Estruturação dos } \\
\text { relatórios e padrões } \\
\text { de alerta }\end{array}$} & \\
\hline & \multirow{2}{*}{$\begin{array}{l}\checkmark \text { Painel de } \\
\text { monitoramento e } \\
\text { controle }\end{array}$} & & \\
\hline & & $\begin{array}{l}\checkmark \text { Testes de } \\
\text { funcionamento }\end{array}$ & \\
\hline & \multirow[t]{2}{*}{$\begin{array}{l}\checkmark \text { Disseminação aos } \\
\text { transportadores }\end{array}$} & $\begin{array}{l}\checkmark \text { Homologação do } \\
\text { sistema }\end{array}$ & \\
\hline & & $\begin{array}{l}\checkmark \text { Treinamento dos } \\
\text { membros envolvidos }\end{array}$ & \\
\hline
\end{tabular}

Figura 2 - Fluxo de atividades na implantação do CCL

Nas etapas de iniciação e planejamento foram realizados trabalhos desde levantamento de demanda até a definição de uma tecnologia que fosse capaz de 
fornecer informações necessárias para compor os KPI's relevantes para gestão eficaz da operação de logística.

Nas etapas posteriores de implantação da ferramenta, houve envolvimento dos transportadores na adesão à tecnologia de rastreamento escolhida, que por meio de uma interface sistêmica gera informações mutuas para Gerdau e seus parceiros.

O sistema de rastreamento escolhido, permite além rastreamento on-line de veículos, avaliar o desempenho da operação em tempo real, onde são monitorados os pontos de carga e descarga, bem como possíveis gargalos na operação. Este monitoramento é realizado a partir de um georreferenciamento, onde são criadas micro e macro cercas que representam os pontos de controle, conforme demonstrado na figura 3.

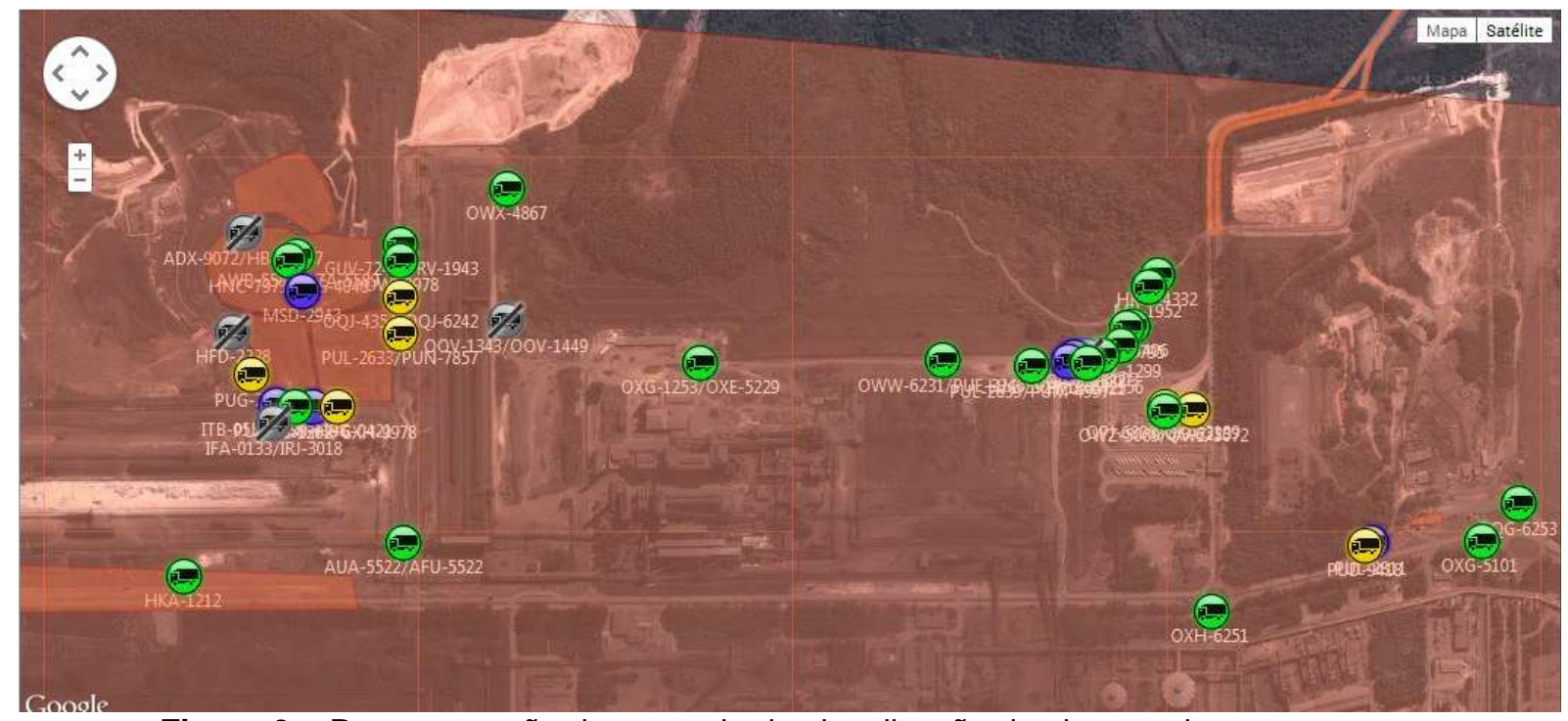

Figura 3 - Representação de uma tela de visualização do sistema de rastreamento

As macro cercas apresentadas graficamente por cores mais claras representam as áreas de fronteira de uma unidade, mina ou usina na qual são movimentados o minério, enquanto as micro cercas apresentadas graficamente por cores mais escuras representam as áreas específicas de carga, descarga, acesso, pesagem, ou qualquer outro ponto que se julgue necessário monitorar.

A partir do sistema de rastreamento são captados dados de movimentação dos veículos que estão em operação na movimentação do minério de ferro Gerdau e transformados em informações relevantes para controle e gestão da operação logística.

\subsubsection{Resultados obtidos com a implantação do CCL}

Com as informações apuradas a partir dos dados do sistema de rastreamento, foi possível construir os chamados painéis de controle e gestão divididos em:

- Painel de movimentações: onde são apontados com interface ao ERP os volumes movimentados entre os pontos de produção e consumo de minério, exemplificado pelo modelo apresentado na figura 4. 


\begin{tabular}{|c|c|c|c|c|c|c|c|c|c|c|}
\hline \multicolumn{11}{|c|}{ OLUME TOTAL CONSOLIDADO } \\
\hline Real Acum & Pex Acum & Plano Acum & & & & & $\Delta \mathbf{P I}$ & & PEX Total & Plano Total \\
\hline$x x x x$ & $x x x x$ & $x x x x$ & $x x x x$ & $\%$ & (:) & $x x x x$ & $\%$ & (:) & $x x x x$ & $x x x x$ \\
\hline
\end{tabular}

Movimentação Rodoviária Total - Logística Operacional Mfe

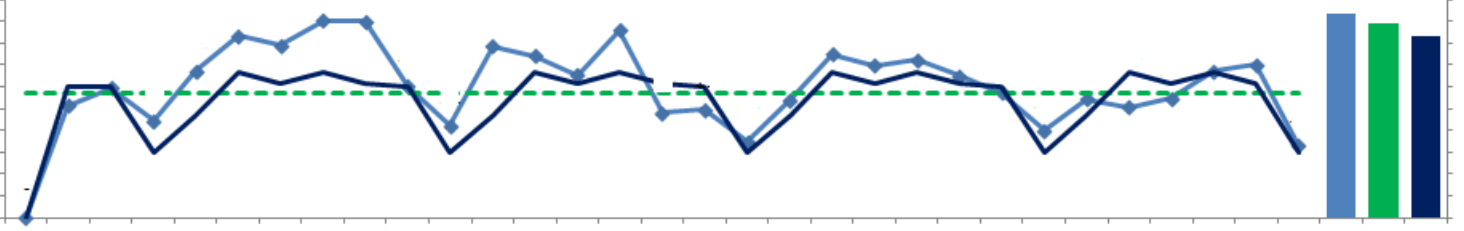

Real Acumulado PEXAcumulado Plano Acumulado $\longrightarrow$ Real $-\infty$ PEX Plano

Figura 4 - Representação gráfica de um dos painéis de movimentação

- Painel de TTP: onde são apontados os tempos de permanência de veículos em cada ponto demarcado por uma geocerca, que consolidados no tempo total de permanência (TTP) da unidade, conforme demonstrado na figura 5.

\begin{tabular}{|l|c|cc|}
\multicolumn{3}{c}{ PAINEL TTP } \\
\cline { 2 - 4 } & Referência & \multicolumn{2}{c|}{ Acumulado Mês } \\
\hline Tempo Total de Permanência & $00: 01: 00$ & 00:01:00 & $\square$ \\
\hline 1. Tempo Médio Descarga & $00: 01: 00$ & $00: 00: 00$ & $\square$ \\
2. Tempo Médio C. Acesso & $00: 02: 00$ & $00: 01: 00$ & $\square$ \\
3. Tempo Médio de Carregamento & $00: 03: 00$ & $00: 04: 00$ & ■ \\
4. Tempo Médio de Pesagem & $00: 04: 00$ & $00: 05: 00$ & ■ \\
5. Tempo Médio de Trânsito Interno / Filas & $00: 06: 00$ & $00: 06: 00$ & $\square$ \\
\hline
\end{tabular}

Figura 5 - Representação gráfica de um painel de TTP

- Painel de capacidades: a partir dos painéis anteriores um painel de capacidades é alimentado de forma dinâmica para nortear as tomadas de decisão e prioridades operacionais, conforme demonstrado na figura 6.

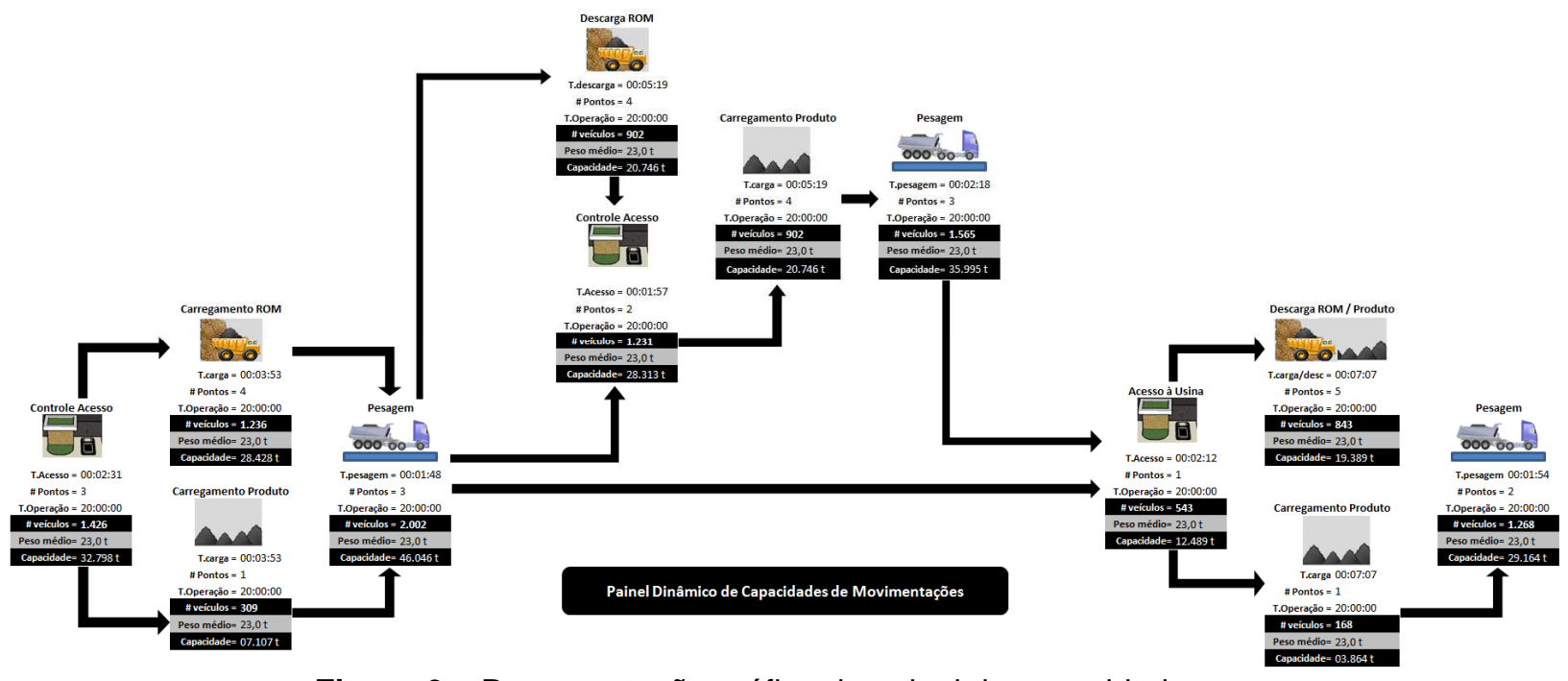

Figura 6 - Representação gráfica do painel de capacidades 
Estes painéis por sua vez consolidam os KPls para gestão logística da operação de minério de ferro da Gerdau.

Além os painéis, outros resultados obtidos com a implantação do CCL foram:

- Controle total da operação terceirizada: a partir de todo o fluxo de informações, a Gerdau tem a possibilidade de fazer gestão completa do desempenho das transportadoras contratadas, em uma operação de alta complexidade;

- Alertas on-line: o CCL permitiu um controle em tempo real das movimentações, possibilitando recebimento de alertas para situações de anormalidade quer são previamente parametrizadas;

- Programação e diligenciamento: o CCL permitiu uma maior eficiência nas programações, alocação de frota e revisão dos planos de entregas, bem como maior eficiência no diligenciamento;

Neste sentido, foi possível desenvolver um conceito amplo de controle e gestão através de uma ferramenta, organizada de forma simples permitindo a gestão de a operações de logística do minério de ferro Gerdau.

\section{CONCLUSÃO}

Os objetivos com a implantação do CCL na operação logística do minério ferro Gerdau foram atingidos em sua totalidade, sendo criada uma base de informações única que consolidada as principais informações relevantes para a gestão e controle de todo o fluxo de movimentação rodoviária do minério de ferro, possibilitando controle da frota, mesmo esta sendo terceirizada.

Além disso, pode-se afirmar que uma sinergia entre o transportador rodoviário e a Gerdau foi um dos fatores de sucesso na implantação da ferramenta, tendo como fundamental a troca mútua de conhecimento das operações de cada uma das partes, bem como as restrições impostas a cada elo desta cadeia.

Além disso, a fundamentação de uma base para efetuar avaliações de desempenho nas prestações de serviço de transporte foi outro ponto fundamental possibilitado a partir da implantação do CCL.

E por fim, a consolidação dos KPis através dos painéis permitiu uma visão geral de toda a operação logística do minério de ferro na Gerdau de forma a enxergar as oportunidades e restrições do sistema, serviço de inputs tanto para projetos de melhoria e otimização de capacidade, como melhorar a alocação dos recursos disponíveis frente as demandas atuais do sistema como um todo.

\section{REFERÊNCIAS}

1 BOWERSOX, Donald J.; CLOSS, David J. - Logistical Management: the Integrated Supply Chain Process - McGraw-Hill - New York: 2001

2 ANEFALOS, L. C. Gerenciamento de Frotas do transporte Rodoviários Utilizado Sistemas de Rastreamento por Satélite. Dissertação de Mestrado em Economia Aplicada, Escola Superior de Agricultura Luiz Queiroz - Piracicaba, 134p.: Il 1999.

3 CAMARA, S. M.; AMORIM, C. H. G. DE A Utilização do Sistema de Rastreamento de Veículos no Transporte de Cargas: Um Estudo de Caso à Segsat. Disponível em: <http://www.simpep.feb.unesp.br/anais/anais_13/artigos/524.pdf> Acessado em 18 fev. 2015.

4 MONICO, João Francisco Galera - Posicionamento pelo GNSS: descrição, fundamentos e aplicações - Editora UNESP. São Paulo: 2008.

5 PRADO, J.; GRAEML, A. R.; PEINADO, J. Percepção dos Benefícios do uso de Sistemas de Rastreadores de Veículos pelos Transportes Disponível em: 
<http://www.dainf.ct.utfpr.edu.br/ graeml/publica/artigos/download/SIMPEP2008_Rastre adores.pdf> Acessado em: 20 de fev. 2015.

6 LIMA Jr.; Caixeta-Filho; Martins. Gestão Logística do Transporte de Cargas - Editora Atlas - São Paulo: 2001.

7 NOVAES, Antônio Galvão. Logística e Gerenciamento da Cadeia de Distribuição: Estratégia, Operação e Avaliação - Editora Campus - Rio de Janeiro: 2001. 